S.-A. GAO

KODAI MATH. $J$.

19 (1996), 355-364

\title{
SOME RESULTS ON THE COMPLEX OSCILLATION FOR HIGHER ORDER HOMOGENEOUS LINEAR DIFFERENTIAL EQUATIONS*
}

\author{
SHI-AN GAO
}

\begin{abstract}
In this paper, we are concerned with the maximum number of linearly independent transcendental solutions with finite exponent of convergence of the zeros for a higher order homogeneous linear differential equation where its coefficients are entire functions with order less than $1 / 2$ and one dominant. The results obtained here are the extension and deepening of $\mathrm{J} . \mathrm{K}$. Langley's.
\end{abstract}

\section{$\S 1$. Introduction and results}

Since 1982, various researchs have been made concerning the complex oscillation of second-order homogeneous linear differential equations. In 1991, J. K. Langley and S. Bank ([2]) discovered surprisingly that some main results obtained from second-order equations still hold for higher-order equations. They made use of asymptotic methods far different to those applied in the secondorder case.

For second-order equations, S. Bank and I. Laine proved the following theorem in [1], in which etc. the notations are defined at the end of this section :

THEOREM A. Suppose that $A(z)$ is a transcendental entire function with $\sigma(A)<1 / 2$. Then, the equation

$$
f^{\prime \prime}+A f=0
$$

can not have two linearly independent solutions $f_{1}, f_{2}$ with $\max \left\{\lambda\left(f_{1}\right), \lambda\left(f_{2}\right)\right\}<+\infty$.

The assertion of Theorem $\mathrm{A}$ has been proved in the case where $\sigma(A)=1 / 2$ by Rossi ([15]) and Shen ([16]) independently.

* Project supported by the National Natural Science Foundation of China.

Keywords: value distribution, differential equation, complex oscillation

AMS No. : 34A20, 30D35.

Received October 2, 1995 ; revised May 20, 1996. 
For higher-order equations, J.K. Langley and S. Bank proved a related conclusion in [2]:

THEOREM B. Suppose that $k \geqq 3$ and that $A_{0}, \cdots, A_{k-2}$ are entire functions such that:

(i) $A_{0}$ is transcendental with $\sigma\left(A_{0}\right)<1 / 2$;

(ii) If $\sigma\left(A_{0}\right)>0$, then $\sigma\left(A_{\jmath}\right)<\sigma\left(A_{0}\right)$ for $j=1, \cdots, k-2$, while if $\sigma\left(A_{0}\right)=0$ then $A_{1}, \cdots, A_{k-2}$ are polynomials.

Then the equation

$$
f^{(k)}+A_{k-2} f^{(k-2)}+\cdots+A_{1} f^{\prime}+A_{0} f=0
$$

can not have two linearly independent solutıons $f_{1}, f_{2}$ with $\max \left\{\lambda\left(f_{1}\right), \lambda\left(f_{2}\right)\right\}<+\infty$.

Afterwards, Langley ([14]) obtained a result with weaker conclusion in a more general situation than Theorem B.

THEOREM C. Suppose that $k \geqq 3$ and that $A_{0}, \cdots, A_{k-2}$ are entire functions such that for some $s \in\{1, \cdots, k-2\}$,

(i) $A_{s}$ is transcendental with $\sigma\left(A_{s}\right)<1 / 2$;

(ii) For $\jmath \neq s$, either $A_{\jmath}$ is a polynomial or we have $\sigma\left(A_{j}\right)<\sigma\left(A_{s}\right)$.

Then the equation (1.1) can not have $k$ linearly independent solutions $f_{1}, \cdots, f_{k}$ with $\max \left\{\lambda\left(f_{1}\right), \cdots, \lambda\left(f_{k}\right)\right\}<+\infty$.

Recently, S.-A. Gao and J.-F. Tang ([7]) got a complement of Theorem C:

THeorem D. With the hypotheses of Theorem C, the equation (1.1) has at most $k-s$ linearly independent transcendental solutions $f_{1}, \cdots, f_{k-s}$ with $\max \left\{\lambda\left(f_{1}\right), \cdots, \lambda\left(f_{k-s}\right)\right\}<+\infty$.

The present paper aims to improve the upper bound $k-s$ in Theorem D, and to obtain a conclusion similar to Theorem $\mathrm{B}$ when $A_{0}$ will be replaced with $A_{s}$. In fact, we prove the following

THEOREM 1. Suppose that $k \geqq 4$ and that $A_{0}, \cdots, A_{k-2}$ are entire functions such that for some $s \in\{2, \cdots, k-2\}$,

(i) $A_{s}$ is transcendental with $\sigma\left(A_{s}\right)<1 / 2$;

(ii) For $j \neq s$, either $A$, is a polynomial or we have $\sigma\left(A_{j}\right)<\sigma\left(A_{s}\right)$. Then

(a) An arbitrary fundamental solution set of the equation (1.1) includes at least $k-s(\geqq 2)$ linearly independent transcendental solutions (in fact, with infinite order); 
(b) The equation (1.1) has at most $m=\min \{k-s, s-1\}$ linearly independent transcendental solutions $f_{1}, \cdots, f_{m}$ with $\max \left\{\lambda\left(f_{1}\right), \cdots, \lambda\left(f_{m}\right)\right\}<+\infty$;

(c) If $s=2$, or if $k-1$ and $k-s$ are relatively prime, then the equation (1.1) can not have two linearly independent transcendental solutions $f_{1}, f_{2}$ with $\max \left\{\lambda\left(f_{1}\right), \lambda\left(f_{2}\right)\right\}<+\infty$.

Theorem 1(b) is an improvement of Theorem D. Under the assumptions above, Theorem 1 (c) is the expected result similar to Theorem B. But the case $s=1$ is exceptional and it remains open here whether we have similar improvements in this case.

In this paper, we use the standard notations of Nevanlinna theory, e.g. see [11]. Secondly, we denote by $\sigma(g), \mu(g)$ and $\lambda(g)$, respectively, the order, the lower order and the exponent of convergence of the zeros for a meromorphic function $g(z)$. Thirdly, for a set $E \subset(1,+\infty)$, denote $m_{1}(E)=\int_{1}^{\infty} \chi_{E}(t) d t / t$, $\underline{\log \operatorname{dens}}(E)=\lim _{r \rightarrow \infty}\left\{m_{1}(E \cap(1, r]) / \log r\right\}, \overline{\log \operatorname{dens}}(E)=\varlimsup_{r \rightarrow \infty}\left\{m_{1}(E \cap(1, r]) / \log r\right\}$, where $\chi_{E}(t)$ denotes the characteristic function of the set $E$.

\section{$\S 2$. Lemmas needed for the proof of Theorem 1}

LEMMA 1 ([10]). Let $w$ be a transcendental meromorphic function with finite order $\boldsymbol{\sigma}(w)=\rho<+\infty$, let $\Gamma=\left\{\left(k_{1}, \jmath_{1}\right), \cdots,\left(k_{m}, \jmath_{m}\right)\right\}$ denote a finite set of distinct pairs of integers that satisfy $k_{\imath}>\jmath_{\imath} \geqq 0$ for $\imath=1, \cdots, m$, and let $\varepsilon>0$ be $a$ given constant. Then there exists a set $E_{1} \subset(1,+\infty)$ with $m_{1}\left(E_{1}\right)<+\infty$, such that for all $z$ satisfying $|z| \notin E_{1} \cup[0,1]$ and $(k, j) \in \Gamma$, we have

$$
\left|w^{(k)}(z) / w^{(\jmath)}(z)\right| \leqq|z|^{(k-\jmath)(\rho-1+\varepsilon)} .
$$

LEMma 2 ([3]). Let $f(z)$ be entire of order $\sigma(f)=\rho<1 / 2$. Denote $A(r)=$ $\inf _{|z|=r} \log |f(z)|, B(r)=\sup _{|z|=r} \log |f(z)|$. If $\rho<\alpha<1$, then

$$
\underline{\log \text { dens }}\{r: A(r)>(\cos \pi \alpha) B(r)\} \geqq 1-\rho / \alpha \text {. }
$$

LEMMA 3 (Theorem 5 of [4]). Let $f(z)$ be entire with $\mu(f)=\mu<1 / 2$ and $\mu<\rho=\sigma(f)$. If $\mu \leqq \delta<\min (\rho, 1 / 2)$ and $\delta<\alpha<1 / 2$, then $\overline{\log \operatorname{dens}}\{r: A(r)>$ $\left.(\cos \pi \alpha) B(r)>r^{\delta}\right\}>C(\rho, \delta, \alpha)$, where $C(\rho, \delta, \alpha)$ is a positive constant depending only on $\rho, \delta$ and $\alpha$.

LEMMA 4 ([6], p. 205). If $f(z)$ is a non-trivial solution of (1.1), where $A_{0}$, $\cdots, A_{k-2}$ are entire functions of finite order, then there is a positive constant $N$ such that

$$
\log |f(z)| \leqq \exp \left(|z|^{N}\right)
$$


LEMMA 5. Suppose that $k \geqq 3$ and that $A_{0}, \cdots, A_{k-2}$ are entire functions of order less than $1 / 2$ such that for some $s \in\{1, \cdots, k-2\}$,

(i) $A_{s}$ is transcendental;

(ii) For $j \neq s$, either $A$, is a polynomial or we have $\sigma\left(A_{\jmath}\right)<\sigma\left(A_{s}\right)$.

If the equation (1.1) has a solution $g$ of finite order, then $g$ is a polynomial.

Proof. If $\sigma\left(A_{s}\right)>0$ then we take $\sigma, \tau$ such that for $j \neq s$,

$$
\boldsymbol{\sigma}\left(A_{j}\right)<\boldsymbol{\sigma}<\tau<\boldsymbol{\sigma}\left(A_{s}\right) .
$$

By Lemma 2 (if $\mu\left(A_{s}\right)=\sigma\left(A_{s}\right)$ ) or by Lemma 3 (if $\mu\left(A_{s}\right)<\sigma\left(A_{s}\right)$ ) we know that there exists a set $H \subset(1,+\infty)$ with $m_{1}(H)=+\infty$, such that for all $z$ satisfying $|z|=r \in H$, we have

$$
\log \left|A_{s}(z)\right|>r^{\tau} .
$$

If $\sigma\left(A_{s}\right)=0$ (thus $\left.\mu\left(A_{s}\right)=\sigma\left(A_{s}\right)=0\right)$, by Lemma 2 there also exists a set $H \subset(1,+\infty)$ with $m_{1}(H)=+\infty$, for $r \in H$

$$
\frac{\min \left\{\log \left|A_{s}(z)\right|:|z|=r\right\}}{\log r} \rightarrow+\infty
$$

as $r \rightarrow+\infty$. For convenience later on, we define $\sigma$ to be zero if $\sigma\left(A_{s}\right)=0$, so that we will always have, if $j \neq s$ and $z$ is large,

$$
\left|A_{j}(z)\right|=O\left(r^{M_{1}} \exp \left(r^{\sigma}\right)\right), \quad M_{1}>0
$$

(Denote some fixed positive constants by $M_{1}, M_{2}, \cdots$ henceforth). If $g$ is a solution of finite order of (1.1) and $g$ is transcendental, by Lemma 1 , there exists $H_{1} \subset(1,+\infty)$ with $m_{1}\left(H_{1}\right)<+\infty$, for all $z$ satisfying $|z|=r \notin H_{1}$,

$$
\left|g^{(j)}(z) / g(z)\right| \leqq|z|^{M_{2}},
$$

for $j=1, \cdots, k$. By (1.1) we obtain

$$
g^{(k)} / g+A_{k-2} g^{(k-2)} / g+\cdots+A_{s} g^{(s)} / g+\cdots+A_{0}=0 .
$$

By (2.7), (2.8) and (2.9) we can get when $|z|=r \notin H_{1}$,

$$
\left|A_{s} g^{(s)} / g\right|=O\left(r^{M_{3}} \exp \left(r^{\sigma}\right)\right) \text {. }
$$

And by (2.5), (2.6) and (2.10), for all $z$ satisfying $|z|=r \in H \backslash H_{1}$,

$$
\left|z^{s} g^{(s)} / g\right|=o(1) \text {. }
$$

On the other hand, by Wiman-Valiron theory (e.g. see [12], [13]), there exists $H_{2} \subset(1,+\infty)$ with $m_{1}\left(H_{2}\right)<+\infty$, such that if $z$ satisfies $|z|=r \notin H_{2}$ and $|g(z)|$ $=M(r, g)$, then $g^{(s)}(z) / g(z) \sim(\nu(r) / z)^{s}$, where $\nu(r)$ is the central index of $g$. From (2.11) we get $\nu(r)=o(1)$ when $r \in H \backslash\left(H_{1} \cup H_{2}\right)$. Since $g$ is transcendental, 
this is a contradiction. So $g$ must be a polynomial.

The following Lemma 6 (see [13, p. 244]) is an improvement of a result of M. Frei ([5], [6]):

LEMMA 6. Suppose that the coefficients of the linear differential equation

$$
a_{n} w^{(n)}+a_{n-1} w^{(n-1)}+\cdots+a_{0} w=0
$$

are entire functions, at least one of $a_{j}(0 \leqq j \leqq n-1)$ is transcendental, and $a_{p}$ $(0 \leqq p \leqq n-1)$ is the first one in order of $a_{0}, a_{1}, \cdots, a_{n-1}$ such that

$$
\varliminf_{\substack{r \rightarrow \infty \\ r \in D}}\left\{\left(\sum_{j=p+1}^{n} m\left(r, a_{j}\right)\right) / m\left(r, a_{p}\right)\right\}<1,
$$

where $D$ is some set in $(0,+\infty)$ with finite measure. Then the equation (2.12) has at most $p$ linearly independent meromorphic solutions with the property

$$
\varlimsup_{\substack{r \rightarrow \infty \\ r \notin D}}\left\{\log T(r, w) / m\left(r, a_{p}\right)\right\}=0 \text {. }
$$

\section{$\S 3$. Proof of Theorem 1}

We first prove part (a) of Theorem 1 .

It is well-known that if $A_{0}, \cdots, A_{k-1}$ are entire functions, then all solutions of the equation (1.1) are entire. As the proof in Lemma 5, by (2.4), (2.5), (2.6) and (2.7), it is easy to check that for an arbitrary set $D$ of $r$ with finite measure we have

$$
\lim _{\substack{r \rightarrow \infty \\ r \notin D}}\left\{\left(\sum_{j=s+1}^{k} m\left(r, A_{\jmath}\right)\right) / m\left(r, A_{s}\right)\right\} \leqq \lim _{\substack{r \rightarrow \infty \\ r \in \boldsymbol{H} \backslash D}}\left\{\left(\sum_{j=s+1}^{k} m\left(r, A_{j}\right)\right) / m\left(r, A_{s}\right)\right\}<1,
$$

i.e. (2.13) holds for the equation (1.1). Additionally, since $A_{s}$ is transcendental, it is easy to see that for an arbitrary polynomial solution $g(z)$, we have

$$
\lim _{r \rightarrow \infty}\left(\log m(r, g) / m\left(r, A_{s}\right)\right)=0,
$$

i.e. (2.14) holds. Therefore, it follows that from Lemma 6 the equation (1.1) has at most $s$ linearly independent polynomial solutions. This implies assertion (a) holds from Lemma 5.

Now, we start to prove part (b) and part (c).

Suppose that the equation (1.1) has $q$ linearly independent transcendental solutions $f_{1}, \cdots, f_{q}$ each with $\lambda\left(f_{\jmath}\right)<+\infty$. Thus, $f_{j}$ can be written in the form $f_{\jmath}=w_{j} e^{h_{\jmath}}$ with $\sigma\left(w_{j}\right)<+\infty, \jmath=1, \cdots, q$. By Lemma $5, \sigma\left(f_{j}\right)=+\infty$, so $h$, must be a transcendental entire function. But by Lemma $4, \sigma\left(h_{j}\right)<+\infty$. Therefore, by Lemma 1 , there exists a set $E_{2} \subset(1,+\infty)$ with $m_{1}\left(E_{2}\right)<+\infty$, for $|z| \notin E_{2} \cup$ $[0,1], m=1, \cdots, k$ and $\jmath=1, \cdots, q$,

$$
\left|w_{j}^{(m)} / w_{\jmath}\right|+\left|h_{j}^{(m)} / h_{j}^{\prime}\right|+\sum_{\imath \neq \jmath}\left|\left(w_{j} / w_{\imath}\right)^{(m)} /\left(w_{j} / w_{\imath}\right)^{\prime}\right| \leqq|z|^{M_{4}}
$$


If $\sigma\left(A_{s}\right)>0$, for $\jmath \neq s$ we take $\sigma, \tau$ such that

$$
\boldsymbol{\sigma}\left(A_{j}\right)<\boldsymbol{\sigma}<\tau<\boldsymbol{\sigma}\left(A_{s}\right) .
$$

By the same reasoning as in the proof of Lemma 5, there exists a sequence $\left\{r_{n}\right\}, r_{n} \rightarrow \infty$, such that $r_{n} \notin E_{2}$ and such that on $|z|=r_{n}$ we have

$$
\left\{\begin{array}{l}
\log \left|A_{s}(z)\right|>r_{n}^{\tau}, \quad \text { if } \sigma\left(A_{s}\right)>0 ; \\
\frac{\min \left\{\log \left|A_{s}(z)\right|:|z|=r_{n}\right\}}{\log r_{n}} \rightarrow+\infty, \quad \text { if } \sigma\left(A_{s}\right)=0,
\end{array}\right.
$$

and moreover, for $j \neq s$, by defining $\sigma=0$ when $\sigma\left(A_{s}\right)=0$, we have always

$$
\left|A_{j}(z)\right|=O\left(|z|^{M_{5}} \exp \left(|z|^{\sigma}\right)\right) \text {. }
$$

Now we estimate $h_{j}^{\prime}$ on $|z|=r_{n}$. By (3.1), there exists $N>0$ ( $N$ can be taken large enough) such that if $z$ on $|z|=r_{n}$ and $\left|h_{j}^{\prime}(z)\right| \geqq|z|^{N}$ then we have

$$
f_{j}^{(p)}(z) / f_{j}(z)=\left(h_{\jmath}^{\prime}\right)^{p}\left(1+O\left(|z|^{-M_{6}}\right)\right),
$$

where $p=1, \cdots, k$. Substituting $f_{\jmath}=w_{j} e^{h_{3}}$ into (1.1) and dividing through by $f_{\jmath}$, at a point $z$ on $|z|=r_{n}$ satisfying $\left|h_{j}^{\prime}(z)\right| \geqq|z|^{N}$, by (3.4) and (3.5) we have

$$
\begin{aligned}
& \left(h_{j}^{\prime}\right)^{k}(1+o(1))+A_{s}\left(h_{\jmath}^{\prime}\right)^{s}(1+o(1))+\sum_{\substack{m=0 \\
(m \neq s)}}^{k-2} O\left(r_{n}^{M_{5}} \exp \left(r_{n}^{\sigma}\right)\right)\left(h_{j}^{\prime}\right)^{m}=0, \text { or } \\
& \left(\left(h_{j}^{\prime}\right)^{k-s} / A_{s}\right)(1+o(1))+1+\sum_{\substack{m=0 \\
(m \neq s)}}^{k-2} O\left(r_{n}^{M_{5}} \exp \left(r_{n}^{\sigma}\right)\right)\left(h_{j}^{\prime}\right)^{m-k}\left(h_{j}^{\prime}\right)^{k-s} / A_{s}=0, \text { or } \\
& \left(\left(h_{j}^{\prime}\right)^{k-s} / A_{s}\right)(1+o(1))+1+\left(\left(h_{j}^{\prime}\right)^{k-s} / A_{s}\right) O\left(r_{n}^{M_{5}} \exp \left(r_{n}^{\sigma}\right)\right) /\left(h_{j}^{\prime}\right)^{2}=0, \text { or } \\
& \left(\left(h_{j}^{\prime}\right)^{k-s} / A_{s}\right)\left(1+O\left(r_{n}^{M_{5}} \exp \left(r_{n}^{\sigma}\right)\right) /\left(h_{j}^{\prime}\right)^{2}\right)=-1 .
\end{aligned}
$$

If there are infinitely many $n$, say $n_{k}$, such that at a point $z$ on $|z|=r_{n_{k}}$ satisfying $\left|h_{j}^{\prime}(z)\right| \geqq|z|^{N}$, we have $\left|h_{j}^{\prime}(z)\right| \leqq \exp \left(r_{n_{k}}^{\sigma}\right)$, then by (3.3), (3.6) can not hold as $n_{k}$ is large enough (because the left side of (3.6) tends to zero as $\left.n_{k} \rightarrow \infty\right)$. So if $n$ is large enough, at a point $z$ on $|z|=r_{n}$ satisfying $\left|h_{j}^{\prime}(z)\right| \geqq$ $|z|^{N},\left|h_{j}^{\prime}(z)\right|>\exp \left(r_{n}^{\sigma}\right)$ must hold. Thus, at a point $z$ on $|z|=r_{n}$ satisfying $\left|h_{j}^{\prime}(z)\right| \geqq|z|^{N}$, from (3.6) we can get

$$
\left(h_{j}^{\prime}\right)^{k-s} / A_{s}=-1+o(1),
$$

and

$$
A_{s} /\left(h_{j}^{\prime}\right)^{k-s}=-\left(1+O\left(r_{n}^{M_{5}}\right) / h_{j}^{\prime}\right) .
$$

Now set $G_{1}=\left\{z:\left|h_{j}^{\prime}(z)\right| \geqq|z|^{N},|z|=r_{n}\right\}, G_{2}=\left\{z:\left|h_{j}^{\prime}(z)\right|<|z|^{N},|z|=r_{n}\right\}, \quad G=$ $\left\{z:|z|=r_{n}\right\}$, then $G=G_{1} \cup G_{2}, G_{1} \cap G_{2}=\emptyset$. By (3.3) and (3.7), it is not difficult to see that $G_{1}=\left\{z:\left|h_{j}^{\prime}(z)\right|>|z|^{N},|z|=r_{n}\right\}$ as $r_{n}$ is large enough. Since the linearly connected open set $G$ can be separated into the union of two open sets 
and $G_{1} \cap G_{2}=\emptyset$, one of the sets $G_{1}$ and $G_{2}$ must be empty. Because $h_{j}^{\prime}(z)$ is a transcendental entire function, so $G_{1} \neq \emptyset$ as $r_{n}$ is large enough, and this is to say $G_{2}=\emptyset$. Therefore, (3.5), (3.6), (3.7) and (3.8) always hold on $|z|=r_{n}$ as $r_{n}$ is large enough.

Using (3.7), from (3.8) we obtain

$$
\begin{aligned}
A_{s}^{1 /(k-s)} / h_{j}^{\prime} & =\left(-1+O\left(r_{n}^{M_{5}}\right) / h_{j}^{\prime}\right)^{1 /(k-s)} \\
& =(-1)^{1 /(k-s)}\left(1+O\left(r_{n}^{M_{5}}\right) / h_{j}^{\prime}\right) .
\end{aligned}
$$

So on $|z|=r_{n}$,

$$
A_{s}^{1 /(k-s)}-(-1)^{1 /(k-s)} h_{j}^{\prime}=O\left(r_{n}^{M_{5}}\right) .
$$

Hence, on $|z|=r_{n}$, there exists $c_{\jmath, n}$ such that $c_{\jmath, n}^{k-s}=-1$ and

$$
\left|h_{j}^{\prime}(z)-c_{j, n} A_{s}(z)^{1 /(k-s)}\right| \leqq r_{n}^{M_{7}} .
$$

Especially,

$$
\left|h_{1}^{\prime}(z)-c_{1, n} A_{s}(z)^{1 /(k-s)}\right| \leqq r_{n}^{M_{7}} .
$$

Therefore, there exists $a_{\jmath, n}$ such that $a_{\jmath, n}^{k-s}=1$ and such that on $|z|=r_{n}$

$$
\left|h_{j}^{\prime}(z)-a_{\jmath, n} h_{1}^{\prime}(z)\right| \leqq r_{n}^{M_{8}} .
$$

Since $a_{\jmath, n}$ is a $(k-s)$-th root of unity, there exists a fixed one, say $a_{\text {, with }}$ $a_{j}^{k-s}=1$, which makes (3.10) to hold for infinitely many $n$. Thus, $h_{j}^{\prime}(z)-a_{j} h_{1}^{\prime}(z)$ is a polynomial and so is $h_{j}(z)-a_{j} h_{1}(z)$. Set $h_{j}(z)-a_{j} h_{1}(z)=P_{j}(z)$, then $f_{j}=$ $w_{j} e^{h_{\jmath}}=w_{j} e^{P_{j}} \cdot e^{a_{j} h_{1}}$. Since the term $e^{P_{j}}$ with $P_{\jmath}$ a polynomial may be incorporated into $w_{\jmath}$, we conclude that without loss of generality $h_{j}(z)=a_{j} h_{1}(z)$, $j=1, \cdots, q$. Then $f$, can be written in the form

$$
f_{\jmath}=w_{\jmath} e^{a_{j} h_{1}}, \quad \jmath=1, \cdots, q .
$$

As we have known, $a$, is some $(k-s)$-th root of unity, but we will further show that $a$, is also some $(s-1)$-th root of unity. Using (3.7) and (3.3), from (3.6) we can get an improving form of (3.8): On $|z|=r_{n}$,

$$
A_{s} /\left(h_{\jmath}^{\prime}\right)^{k-s}=-\left(1+O\left(r_{n}^{M_{5}}\right) /\left(h_{\jmath}^{\prime}\right)^{1+\varepsilon}\right),
$$

where $0<\varepsilon<1$ is a given constant. In addition, by [11], Lemma 3.5, by (3.1) and by a straightforward inductive argument applied to $f_{j}^{\prime} / f_{j}=h_{j}^{\prime}+w_{j}^{\prime} / w_{j}$ on $|z|=r_{n}$, we get

$$
f_{j}^{(p)} / f_{j}=O\left(r_{n}^{M_{4}}\right)\left(h_{j}^{\prime}\right)^{p-2}+\left(\begin{array}{c}
p \\
1
\end{array}\right)\left(h_{j}^{\prime}\right)^{p-1} w_{j}^{\prime} / w_{j}+\left(\begin{array}{c}
p \\
2
\end{array}\right)\left(h_{j}^{\prime}\right)^{p-2} h_{j}^{\prime \prime}+\left(h_{j}^{\prime}\right)^{p}
$$

for $p=2, \cdots, k$. Moreover, by (3.1) and (3.8)', we may write on $|z|=r_{n}$ 


$$
\begin{aligned}
A_{s} f_{j}^{(s)} / f_{j} & =A_{s}\left[O\left(r_{n}^{M_{4}}\right)\left(h_{j}^{\prime}\right)^{s-2}+\left(\begin{array}{c}
s \\
1
\end{array}\right)\left(h_{j}^{\prime}\right)^{s-1} w_{j}^{\prime} / w_{j}+\left(\begin{array}{c}
s \\
2
\end{array}\right)\left(h_{j}^{\prime}\right)^{s-2} h_{j}^{\prime \prime}+\left(h_{j}^{\prime}\right)^{s}\right] \\
& =O\left(r_{n}^{M}{ }^{M}\right)\left(h_{j}^{\prime}\right)^{k-1-\varepsilon}-\left(\begin{array}{c}
s \\
1
\end{array}\right)\left(h_{j}^{\prime}\right)^{k-1} w_{j}^{\prime} / w_{j}-\left(\begin{array}{c}
s \\
2
\end{array}\right)\left(h_{j}^{\prime}\right)^{k-2} h_{j}^{\prime \prime}-\left(h_{j}^{\prime}\right)^{k} .
\end{aligned}
$$

Since $f_{1}$ and $f_{\jmath}$ are solutions of (1.1), we have

$$
f_{1}^{(k)} / f_{1}+\cdots+A_{1} f_{1}^{\prime} / f_{1}=f_{j}^{(k)} / f_{j}+\cdots+A_{1} f_{j}^{\prime} / f_{\jmath} .
$$

From it and by (3.4), (3.5), on $|z|=r_{n}$, we can get

$$
\begin{aligned}
& f_{1}^{(k)} / f_{1}+A_{s} f_{1}^{(s)} / f_{1}+\sum_{\substack{m=1 \\
(m \neq s)}}^{k-2} O\left(r_{n}^{M_{5}} \exp \left(r_{n}^{\sigma}\right)\right)\left(h_{1}^{\prime}\right)^{m} \\
= & f_{j}^{(k)} / f_{j}+A_{s} f_{j}^{(s)} / f_{j}+\sum_{\substack{m=1 \\
(m \neq s)}}^{k-2} O\left(r_{n}^{M_{5}} \exp \left(r_{n}^{\sigma}\right)\right)\left(h_{j}^{\prime}\right)^{m} .
\end{aligned}
$$

By (3.12), (3,13), (3.11), (3.7), (3.3) and (3.2), dividing through by $\left(h_{1}^{\prime}\right)^{k-1}$, the above formula can be changed into the form on $|z|=r_{n}$

$$
\begin{aligned}
& 2(k-s) w_{1}^{\prime} / w_{1}+[k(k-1)-s(s-1)] h_{1}^{\prime \prime} / h_{1}^{\prime} \\
= & a_{j}^{k-1}\left\{2(k-s) w_{j}^{\prime} / w_{j}+[k(k-1)-s(s-1)] h_{1}^{\prime \prime} / h_{1}^{\prime}\right\}+O\left(r_{n}^{M_{10}}\right)\left(h_{1}^{\prime}\right)^{-\varepsilon} .
\end{aligned}
$$

Denote by $F_{1}$ and $F_{3}$ respectively the entire functions $w_{1}^{2(k-s)}\left(h_{1}^{\prime}\right)^{k(k-1)-s(s-1)}$ and $w_{j}^{2(k-s)}\left(h_{1}^{\prime}\right)^{k(k-1)-s(s-1)}$. By (3.9), $h_{1}^{\prime}$ has lower order less than $1 / 2$, so that $F_{1}$ and $F$, have infinitely many zeros. But the argument principle and (3.14) give

$$
n\left(r_{n}, 1 / F_{1}\right)=a_{j}^{k-1} n\left(r_{n}, 1 / F_{j}\right)+O\left(r_{n}^{M_{10}}\right) M\left(r_{n},\left(h_{1}^{\prime}\right)^{-\varepsilon}\right) .
$$

Since, by (3.7) and (3.3), $O\left(r_{n}^{M_{10}}\right) M\left(r_{n},\left(h_{1}^{\prime}\right)^{-\varepsilon}\right) \rightarrow 0$ as $n \rightarrow \infty$ on $|z|=r_{n}$, the above formula implies that $a_{0}^{k-1}=1$, i.e. $a_{3}$ is also a $(k-1)$-th root of unity. And from $1=a_{\jmath}^{k-1}=a_{\jmath}^{k-s+s-1}=a_{\jmath}^{s-1}$, we see that $a_{\jmath}$ is also a $(s-1)$-th root of unity.

Since $a$, is of above properties, it follows that: 1. In part (b), if $q>\min \{k-s, s-1\}$, then there must exist $i$ and $p, i \neq p$, such that $a_{\imath}=a_{p}$. Thus, by (3.11) we have $h_{i}=h_{p}$. 2. In part (c), assume $q=2$. Since $s=2$ or $k-1$ and $k-s$ are relatively prime, $a,=1$ must hold for $j=1,2$. Therefore, in both cases, there are two transcendental solutions, which may be denoted by $f_{1}$ and $f_{2}$ in Case 1 without loss of generality, of the form $f_{1}=w_{1} e^{h}, f_{2}=w_{2} e^{h}$, where we set $h=h_{1}=h_{2}$. We proceed to show that this is impossible, which proves part (b) and part (c).

Set $f=u f_{1}, v=u^{\prime}$, and substitute them into (1.1), we obtain

$$
v^{(k-1)}+d_{k-2} v^{(k-2)}+\cdots+d_{0} v=0,
$$


where

$$
\left\{\begin{aligned}
d_{k-2}= & k f_{1}^{\prime} / f_{1} \\
d_{k-3}= & A_{k-2}+\left(\begin{array}{c}
k \\
2
\end{array}\right) f_{1}^{\prime \prime} / f_{1} \\
& \ldots \ldots \\
d_{\jmath}= & A_{\jmath+1}+\left(\begin{array}{c}
j+2 \\
1
\end{array}\right) A_{\jmath+2} f_{1}^{\prime} / f_{1}+\cdots+\left(\begin{array}{c}
j+i \\
i-1
\end{array}\right) A_{\jmath+2} f_{1}^{(i-1)} / f_{1}+\cdots \\
& +\left(\begin{array}{c}
s \\
s-j-1
\end{array}\right) A_{s} f_{1}^{(s-\jmath-1)} / f_{1}+\cdots+\left(\begin{array}{c}
k \\
k-j-1
\end{array}\right) f_{1}^{(k-\jmath-1)} / f_{1}, \\
& \cdots \cdots \\
d_{0}= & A_{1}+\left(\begin{array}{c}
2 \\
1
\end{array}\right) A_{2} f_{1}^{\prime} / f_{1}+\cdots+\left(\begin{array}{c}
s \\
s-1
\end{array}\right) A_{s} f_{1}^{(s-1)} / f_{1}+\cdots+k f_{1}^{(k-1)} / f_{1} .
\end{aligned}\right.
$$

Since $f_{1}^{(\jmath)} / f_{1}=\left(h^{\prime}\right)^{\jmath}(1+o(1))(1 \leqq j \leqq k)$ on $|z|=r_{n}$, by (3.3), (3.4), (3.7) and (3.16), we obtain

$$
\left\{\begin{aligned}
d_{0}= & (k-s)\left(h^{\prime}\right)^{k-1}(1+o(1)) \\
d_{1}= & \left(\left(\begin{array}{c}
k \\
k-2
\end{array}\right)-\left(\begin{array}{c}
s \\
s-2
\end{array}\right)\right)\left(h^{\prime}\right)^{k-2}(1+o(1)) \\
& \cdots \cdots \\
d_{s-1}= & \left(\left(\begin{array}{c}
k \\
k-s
\end{array}\right)-\left(\begin{array}{c}
s \\
0
\end{array}\right)\right)\left(h^{\prime}\right)^{k-s}(1+o(1)) \\
& \cdots \cdots \\
d_{k-2}= & \left(\begin{array}{c}
k \\
1
\end{array}\right) h^{\prime}(1+o(1))
\end{aligned}\right.
$$

on $|z|=r_{n}$. Obviously, $v=\left(f_{2} / f_{1}\right)^{\prime}=\left(w_{2} / w_{1}\right)^{\prime}$ is a solution of (3.15). By (3.1), when $|z| \notin E_{2} \cup[0,1]$,

$$
\left|v^{(\jmath)}(z) / v(z)\right| \leqq|z|^{M_{4}}
$$

for $j=1, \cdots, k-1$. Dividing through (3.15) by $v$, we obtain

$$
v^{(k-1)} / v+d_{k-2} v^{(k-2)} / v+\cdots+d_{1} v^{\prime} / v+d_{0}=0 .
$$

Dividing through (3.19) by $\left(h^{\prime}\right)^{k-1}$ again, by (3.3), (3.7), (3.17) and (3.18) on $|z|=r_{n}$, we obtain $k-s=0$ as $r_{n} \rightarrow \infty$. This is a contradiction, since $s \in$ $\{2, \cdots, k-2\}$.

The proof of Theorem 1 has been completed.

Acknowledgement. The author would like to thank the referee for his (or her) serious and valuable comments. 


\section{REFERENCES}

[1] S. Bank AND I. LAINE, On the oscillation theory of $f^{\prime \prime}+A f=0$ where $A$ is entire, Trans. Amer. Math. Soc., 273 (1982), 351-363.

[2] S. BANK AND J.K. LANGLEY, Oscillation theory for higher order linear differential equations with entire coefficients, Complex Variables Theory Appl., 16 (1991), 163-175.

[3] P.D. Barry, On a theorem of Besicovitch, Quart. J. Math. Oxford Ser. (2), 14 (1963), 293-302.

[4] P.D. BARry, Some theorems related to the $\cos \pi \rho$ theorem, Proc. London Math. Soc. (3), 21 (1970), 334-360.

[5] M. FREI, Sur l'order des solutions entières d'une équation différentielle lineare, C. R. Acad. Sci. Paris Ser. I. Math., 236 (1953), 38-40.

[6] M. FREI. Über die Lösungen linearen Differentialgleichungen mit Ganzen Funktionen als Koeffizienten, Comment. Math. Helv., 35 (1961), 201-222.

[7] S.-A. GAO AND J.-F. TANG, A note on the complex oscillation for higher order homogeneous linear differential equations, Ann. Differential Equations, 12 (1996), $167-172$.

[8] S.-A. GAO, Some results on the complex oscillation theory of periodic second order linear differential equations, Kexue Tongbao, 13 (1988), 1064-1068.

[9] S.-A. GAO, A further result on the complex oscillation theory of periodic second order linear differential equations, Proc. Edinburgh Math. Soc., 33 (1990), 143-158.

[10] G. Gundersen, Estimates for the logarithmic derivative of a meromorphic function, plus similar estimates, J. London Math. Soc. (2), 37 (1988), 88-104.

[11] W.K. HAYMan, Meromorphic Functions, Clarendon Press, Oxford, 1964.

[12] W.K. HAYMAN, The local growth of power series: a survey of the WimanValiron method, Canad. Math. Bull., 17 (1974), 317-358.

[13] Y.-Z. HE AND X.-Z. XIAO, Algebraic Functions and Ordinary Differential Equations, Science Press, Peking, 1988 (in Chinese).

[14] J.K. LANGLEY, Some oscillation theorems for higher order linear differential equations with entire coefficients of small growth, Results Math., 20 (1991), 517-529.

[15] J. RossI, Second order differential equations with transcendental coefficients, Proc. Amer. Math. Soc., 97 (1986), 61-66.

[16] L.-C. SHEN, Solutions to a problem of S. Bank regarding the exponent of convergence of the solutions of a differential equation $f^{\prime \prime}+A f=0$, Kexue Tongbao, 30 (1985), 1581-1585.

Department of Mathematics

South China Normal University

GUANGZHOU, 510631

P.R. CHINA 